\title{
Evaluación comparativa de efectividad y tolerabilidad con polietilenglicol y picosulfato de sodio-citrato de magnesio como agentes de preparación intestinal para colonoscopia
}

\author{
Nelson Muñoz P. ${ }^{1}$, Marcelo Rodríguez G. ${ }^{1,2}$, Nicolás Campaña W. ${ }^{3}$, \\ Solange Agar F. ${ }^{1}$ y Gonzalo Campaña V. ${ }^{1,2}$
}

Clínica INDISA

¿Universidad Andrés Bello Campus Clínico INDISA Facultad de Medicina. ${ }^{3}$ CESFAM Dr. Luis Ferrada Urzúa, Maipú. Santiago, Chile.

Recibido el 19 de septiembre aceptado para publicación el 15 de noviembre de 2017

Correspondencia a: Dr. Gonzalo Campaña V. gonzalo.campana@indisa.c
Comparative evaluation of effectiveness and tolerability with polyethylene glycol and sodium picosulfate-magnesium citrate as intestinal preparation agents for colonoscopy

Introduction: The effectiveness of colonoscopy depends on multiple factors, being two of the most important ones an adequate bowel preparation and the patient's tolerability to the preparation. Objectives: Compare effectiveness and tolerability of two bowel preparation agents, polyethylene glycol (PEG) and sodium picosulfate/magnesium citrate (SPMC). Patients and Methods: Randomized clinical trial on outpatients that went into colonoscopy in INDISA Clinic. We evaluated effectiveness and tolerability with Boston Bowel Preparation Score (BBPS) and Lawrence questionnaire [composed by Likert scale, two qualitative questions and Visual Analogue Scale (VAS) for pain], respectively. Results: 189 patients, 123 were randomized to PEG and 66 to SPMC. BBPS average in patients in the PEG branch was 7.51 (SD 1.66) and for SPMC 7.12 (SD 1.71) $(\mathrm{p}=0.111)$. Likert scale for evaluating tolerability average for PEG was 0.94 (SD 0.68) and for SPMC 0.63 (SD 0.61) ( $\mathrm{p}=0.0004)$. VAS scale for PEG had an average of 7.68 (SD 2.4) and for PSCM 9.04 (SD 1.59) ( $\mathrm{p}<0.0001)$. When we asked for workplace absenteeism, there were no significant differences between both groups and when we asked about using the same intestinal preparation in a future colonoscopy there was statistical significance in favor to SPMC $(p=0.026)$. Conclusions: No differences were noted on effectiveness between the PEG and SPMC bowel preparations. Nevertheless, SPMC appeared to be better tolerated by patients.

Key words: bowel preparation solutions; colonoscopy; polyethylene glycols; sodium picosulfate.

\section{Resumen}

Introducción: La efectividad de la colonoscopia depende de múltiples factores, destacando la calidad de preparación intestinal y la tolerabilidad que tenga el paciente a la preparación administrada. Objetivo: Comparar dos agentes de preparación intestinal, el polietilenglicol (PEG) y el picosulfato de sodiocitrato de magnesio (PSCM) en términos de efectividad y tolerabilidad de la preparación. Pacientes y Método: Ensayo clínico aleatorizado en pacientes ambulatorios sometidos a colonoscopia en Clínica INDISA. Evaluando efectividad y tolerabilidad con el Boston Bowel Preparation Score (BBPS) y cuestionario de Lawrence [compuesto por escala Likert, dos preguntas cualitativas y escala visual análoga (EVA)], respectivamente. Resultados: 189 pacientes, de los cuales 123 se aleatorizaron a PEG y 66 a PSCM. El BBPS en los pacientes que utilizaron PEG, la media fue 7,51 (DS 1,66) y con PSCM fue 7,12 (DS 1,71) $(p=0,111)$. Al analizar la tolerabilidad con escala Likert, la media con PEG fue 0,94 (DS 0,68$)$ y con PSCM fue 0,63 (DS 0,61$)(p=0,0004)$. La EVA con PEG tuvo una media de 7,68 (DS 2,4) y con PSCM de 9,04 (DS 1,59) (p<0,0001). Al preguntar ausentismo laboral, no hubo diferencias significativas en ambos grupos y al preguntar si ocuparía la misma preparación en una futura colonoscopia, hubo significancia estadística a favor del PSCM $(p=0,026)$. Conclusión: No encontramos diferencias en la efectividad de preparación intestinal al comparar PEG y PSCM, sin embargo, el PSCM es mejor tolerado. Palabras clave: solución preparación intestinal; colonoscopia; polietilenglicol; picosulfato de sodio. 


\section{Introducción}

En Estados Unidos la tasa de mortalidad del cáncer colorrectal (CCR) fue de 14,8 por cada 100.000 habitantes en el 2014 ${ }^{1,2}$. En Chile, la tasa de mortalidad para 2015 fue de 10,3 y 2,7 por 100.000 habitantes para cáncer de colon y recto respectivamente ${ }^{3}$. Este cáncer en estadios iniciales es asintomático, por tanto, es trascendental su búsqueda activa a través del screening. Se ha demostrado que este tiene un impacto en la mortalidad del CCR, disminuyendo su tasa ${ }^{1}$.

La colonoscopia es el principal método para la evaluación del colon y es de elección para la detección precoz del CCR. Su eficacia diagnóstica y terapéutica depende de varios factores ${ }^{4}$, entre ellos el grado de calidad de la técnica y el equilibrio entre una preparación adecuada y la satisfacción del paciente al procedimiento ${ }^{5}$.

El agente ideal utilizado para la preparación colónica deben tener características tales como eliminar del colon toda materia fecal, paso rápido intestinal para no alterar la mucosa, no provocar alteraciones hidroelectrolíticas, tener un perfil de seguridad alto, que sea bien tolerado y accesible económicamente ${ }^{5}$. Existen diferentes agentes de preparación en el mercado, cada uno de ellos con propiedades, composición y posología heterogéneas ${ }^{6}$. Lamentablemente, ninguna de las opciones con las que contamos actualmente reúne todas estas características.

Una preparación colónica adecuada se define como aquella limpieza del colon que permite una evaluación óptima de la mucosa, visualización de pólipos mayores o iguales a $5 \mathrm{~mm}$ y tasas de intubación cecal altas ${ }^{5,7,8}$.

La preparación subóptima del intestino para la colonoscopia se puede presentar hasta en un $25 \%$. Existe sólida evidencia que una mala preparación intestinal va a afectar directamente la calidad de la colonoscopia y esto disminuye la capacidad de detección de lesiones pequeñas?.

Los agentes de preparación intestinal destinados a la limpieza, previo a la colonoscopia, se evalúan en base a su efectividad, seguridad y tolerabilidad.

La preparación más comúnmente usada es el polietilenglicol (PEG), que es un laxante osmótico con electrolitos añadidos, seguro y altamente efectivo en limpieza y que se ha aceptado como el estándar en preparación colónica ${ }^{5,10}$. Sin embargo, la ingesta de altos volúmenes (4 litros) de PEG es un problema en pacientes con insuficiencia cardiaca, hepática y renal. El sabor desagradable hace que los resultados no siempre sean los esperables en términos de tole- rabilidad. La tasa de limpieza adecuada reportada va entre el $71-92 \%{ }^{8,10,11}$.

El picosulfato de sodio más citrato magnésico (PSCM) es un agente de bajo volumen (2 litros), con acción dual, actuando localmente en el colon como un laxante estimulante al aumentar la frecuencia y la fuerza del peristaltismo, como también un laxante osmótico al retener líquidos en el colon. El PSCM es bien tolerado con una tasa de limpieza entre el $65-85 \%{ }^{8,12}$.

Existen varios ensayos clínicos en la literatura, los cuales comparan la efectividad y tolerabilidad del PEG y PSCM, sin embargo, en zonas geográficas disímiles y con resultados dispares ${ }^{13,21}$.

En nuestra búsqueda permanente para poder darle a nuestros pacientes la mejor preparación colonoscópica y ante la llegada en los últimos años del PSCM con sus características ya mencionadas, es que quisimos evaluar sus resultados en forma local comparándolo con PEG.

El objetivo primario de este estudio es comparar la efectividad del PEG y el PSCM en términos de calidad de preparación intestinal utilizando escala de Boston ${ }^{22,23}$, tasa de intubación cecal y detección de adenomas. El objetivo secundario es evaluar la tolerancia del paciente con ambas preparaciones, según encuesta de Lawrence ${ }^{24}$.

\section{Pacientes y Método}

Se trata de un ensayo clínico aleatorizado ciego simple, llevado a cabo en Unidad de Endoscopia de Clínica INDISA, durante junio de 2017. Los datos recopilados se incluyeron en tabla Excel ${ }^{\circledR}$ con información codificada y anonimizada.

El universo está constituido por pacientes a los que se le realiza colonoscopia en Clínica INDISA. Se realizó cálculo del tamaño muestral utilizando comparación de dos medias, aplicando test bilateral, con un nivel de confianza del $95 \%$, poder estadístico del $80 \%$, precisión de 1,0 y varianza de 4,0 , obteniendo un tamaño muestral de 63 pacientes por rama, ajustando las pérdidas al $10 \%$, obtenemos 70 pacientes por rama.

Se establecieron como criterios de inclusión, pacientes correlativos ambulatorios, ambos sexos, entre 18-70 años, que voluntariamente acepten participar en el estudio firmando consentimiento informado. Se excluyeron aquellos con antecedente de cirugías de colon, enfermedad inflamatoria intestinal, falla cardiaca severa clase III o IV de New York Heart Association (NYHA), cirrosis hepática severa Child Pugh C, insuficiencia renal, embarazadas y colonoscopias de urgencia. 
La aleatorización se realizó mediante muestreo probabilístico aleatorio simple con dígito verificador del registro único tributario (RUT), realizada por el investigador principal, quien no participó en la realización de las colonoscopias.

A los pacientes se les entregaron indicaciones detalladas de preparación vía telefónica y por escrito vía correo electrónico. La indicación de dieta fue iniciar régimen claro desde el día previo. Cada sobre de PEG contiene polietilenglicol 14,97 g, sulfato de sodio 1,40 g, bicarbonato de sodio $0,42 \mathrm{~g}$, cloruro de sodio $0,37 \mathrm{~g}$, cloruro de potasio $0,19 \mathrm{~g}$, bifosfato de sodio 0,12 g y cada sobre de PSCM contiene picosulfato de sodio $0,01 \mathrm{~g}$, óxido de magnesio 3,5 g y ácido cítrico $12 \mathrm{~g}$. Las indicaciones precisas se resumen en Tabla 1.
Para evaluar le eficacia limpiadora se utilizó el Boston Bowel Preparation Score (BBPS), el cual analiza tres segmentos del colon (derecho, transverso e izquierdo) y se clasifica cada segmento según limpieza del 0 al 3, siendo 3 el puntaje óptimo, dando un puntaje final que va del 0 al 9 . Este instrumento está validado y es el recomendado para evaluar la efectividad de las colonoscopias al compararlo con otras escalas $^{23}$. El BBPS (Figura 1) fue medido por los 13 endoscopistas staff participantes en el estudio, en fase de retirada colonoscópica y luego de realizar maniobras de limpieza. Se consideró como una preparación adecuada aquella con BBPS $\geq 6$ con al menos 2 puntos en cada segmento.

Para evaluar la tolerabilidad se utilizó el cuestionario de Lawrence ${ }^{24}$ (Figura 2) el cual consta de

Tabla 1. Indicaciones de esquema y tipo de preparación colonoscópica

\begin{tabular}{|c|c|c|}
\hline $\begin{array}{l}\text { Esquema/ } \\
\text { Tipo preparación }\end{array}$ & $\begin{array}{l}\text { PEG } \\
\text { Diluir } 16 \text { sobres en } 4 \text { litros de agua }\end{array}$ & $\begin{array}{l}\text { PSCM } \\
\text { Diluir } 2 \text { sobres en } 150 \mathrm{ml} \text { de agua cada uno }\end{array}$ \\
\hline $\begin{array}{l}\text { Día previo } \\
\text { (colonoscopia } \\
\text { en la mañana) }\end{array}$ & $\begin{array}{l}2 \text { litros de PEG después de almuerzo y } 2 \text { litros } \\
\text { desde las 20:00, tomando } 250 \mathrm{ml} \text { cada } 10 \mathrm{~min}\end{array}$ & $\begin{array}{l}\text { Primer sobre después de almuerzo seguido de } 250 \mathrm{ml} \text { de agua cada } \\
1 \mathrm{~h} \text { hasta completar } 1.250 \mathrm{cc} \\
\text { Segundo sobre a las } 20: 00 \text { seguido de } 250 \mathrm{ml} \text { de agua cada } 1 \mathrm{~h} \\
\text { por } 3 \text { veces }\end{array}$ \\
\hline $\begin{array}{l}\text { Dosis dividida (Split) } \\
\text { (colonoscopia a } \\
\text { medio día o tarde) }\end{array}$ & $\begin{array}{l}2 \text { litros en la tarde anterior desde las 19:00 y } 2 \\
\text { litros desde las 07:00 del día del examen }\end{array}$ & $\begin{array}{l}\text { Primer sobre desde las 19:00 seguido de } 250 \mathrm{ml} \text { de agua cada } 1 \mathrm{~h} \\
\text { hasta completar } 1.250 \mathrm{cc} \\
\text { Segundo sobre a las 07:00 h seguido de } 250 \mathrm{ml} \text { de agua cada } 1 \mathrm{~h} \\
\text { por } 3 \text { veces hasta } 2 \mathrm{~h} \text { antes del procedimiento }\end{array}$ \\
\hline
\end{tabular}

$250 \mathrm{ml}=1$ vaso grande.

\begin{tabular}{|c|c|c|c|c|}
\hline ESCALA BOSTON & 3 PUNTOS & 2 PUNTOS & 1 PUNTO & 0 PUNTOS \\
\hline $\begin{array}{l}3=\text { EXCELENTE } \\
2=\text { BUENA } \\
1=\text { MALA } \\
0=\text { INADECUADA }\end{array}$ & & & & \\
\hline COLON DERECHO & $\square$ & $\square$ & $\square$ & $\square$ \\
\hline COLON TRANSVERSO & $\square$ & $\square$ & $\square$ & $\square$ \\
\hline COLON IZQUIERDO & $\square$ & $\square$ & $\square$ & $\square$ \\
\hline PUNTAJE FINAL: & & & & \\
\hline
\end{tabular}

Figura 1. Boston Bowel Preparation Score. 3 puntos: excelente visualización de la mucosa, sin presencia de restos líquidos. 2 puntos: escaso contenido fecal líquido que permite una buena visualización de la mucosa. 1 punto: áreas del segmento del colon no vistas por presencia de contenido fecal líquido y semisólido. 0 puntos: segmento de colon no preparado, con mucosa no visualizada por presencia de heces sólidas. 


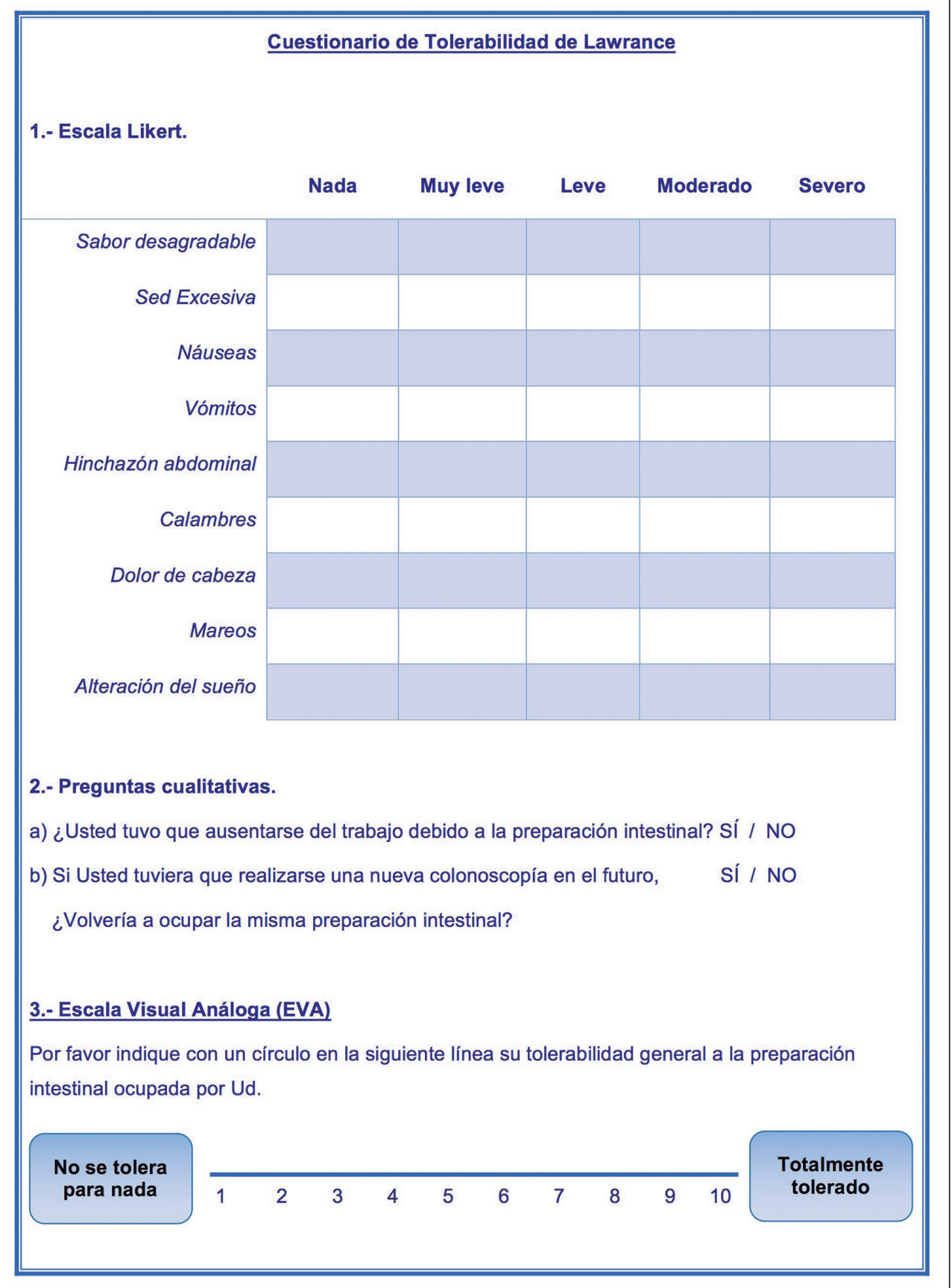

Figura 2. Cuestionario de tolerabilidad de Lawrence. Escala de Likert nada: 0 puntos, muy leve: 1 punto, leve: 2 puntos, moderado: 3 puntos, severo: 4 puntos. Un valor de promedio más bajo indica mejor tolerabilidad.

3 partes: una escala de Likert, dos preguntas cualitativas y escala visual análoga (EVA). La escala de Likert consta de 9 variables referentes a síntomas relacionados a la ingesta del agente de preparación medida cuantitativamente del 0 al 4 en cada una de las variables medidas. Elegimos este cuestionario por ser una encuesta ya validada ${ }^{24}$, su fácil reproducibilidad y rápida aplicación. Los pacientes com- pletan el cuestionario en la sala de precolonoscopia, con asistencia de enfermera entrenada.

Otras variables tales como perfil biodemográfico, indicación de colonoscopia, hallazgos endoscópicos, incluyendo intubación cecal y detección de adenomas, y revisión de biopsias estuvieron a cargo del investigador principal.

Para el análisis estadístico se utilizó estadística 
analítica mediante empleo de software STATA 13.0. El valor de significancia estadística considerado fue $p<0,05$. Se realizó un análisis descriptivo de las características basales. Las variables cuantitativas continuas se expresan en medias \pm DS y variables categóricas se expresan en números y porcentajes. Se utilizó $\chi^{2}$ para variables cualitativas y MannWhitney para variables cuantitativas que no presentan distribución normal.

Este estudio cuenta con la aprobación del Comité de Bioética de la Universidad Andrés Bello, cumpliendo principios éticos de la Declaración de Helsinki y Taipéi ${ }^{25,26}$.

\section{Resultados}

Se enrolaron un total de 280 pacientes, de ellos 16 cumplieron con alguno de los criterios de exclusión; cirugía colónica previa $(\mathrm{n}=5)$, enfermedad inflamatoria intestinal $(\mathrm{n}=3)$, falla cardiaca severa $(n=3)$, cirrosis hepática severa $(n=3)$, insuficiencia renal $(\mathrm{n}=2)$ y hubo otros 7 pacientes que rechazaron participar. Se aleatorizaron 257 pacientes de los cuales 139 fueron a PEG y 117 a PSCM. En la rama del PEG, 12 pacientes no recibieron intervención asignada, 9 por cancelación de colonoscopia y 3 que, posteriormente, rechazaron participar, mientras que en la rama del PSCM, 22 pacientes cancelaron colonoscopia, 15 rechazaron participar posteriormente y 10 pacientes ocuparon una preparación diferente a la indicada. En el análisis se excluyeron 4 pacientes de cada rama por no completar encuesta en su totalidad, quedando finalmente para el análisis 189 pacientes, de los cuales 123 utilizaron PEG y 66 PSCM (Figura 3) ${ }^{27}$. La media de edad fue de 48,5 años, sexo femenino $61,9 \%$, media de IMC 25,4 y ASA I-II 97,9\%. Al comparar ambos grupos en sus características basales, en cuanto a edad, sexo, IMC, ASA e indicaciones del procedimiento $(\mathrm{p}=0,895$, $\mathrm{p}=0,964, \mathrm{p}=0,254 \mathrm{p}=0,712, \mathrm{p}=0,536$ respectivamente) ambos grupos son comparables (Tabla 2 ).

Figura 3. Diagrama de flujo de par-

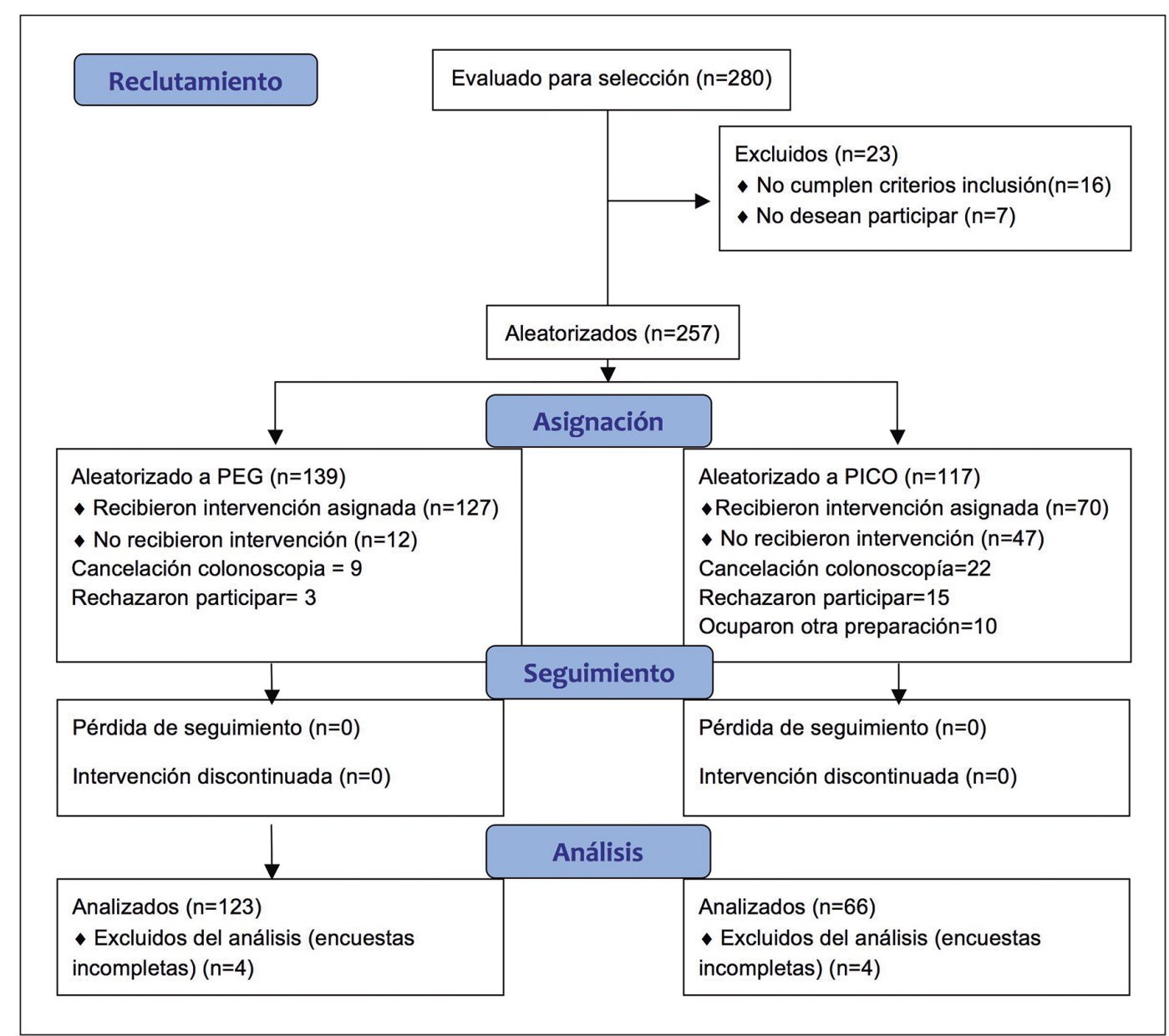


Al analizar el objetivo primario, el BBPS (Tabla 3) como variable continua en los pacientes que utilizaron PEG, la media fue 7,51 (DS 1,66) y con PSCM fue 7,12 (DS 1,71), sin diferencia estadísticamente significativa al aplicar prueba de MannWhitney $(\mathrm{p}=0,1116)$. Si consideramos el BBPS como variable categórica definiendo preparación adecuada aquella con BBPS $\geq 6$ con al menos 2 puntos en cada segmento, tampoco obtenemos diferencias estadísticamente significativas al utilizar $\chi^{2}(\mathrm{p}=0,1167)$.

$\mathrm{Al}$ analizar por tiempo transcurrido entre término de la preparación y el inicio de la colonoscopia estableciendo un corte en $8 \mathrm{~h}$, la media del BBPS fue de 7,98 (DS 1,38), 7,06 (DS 1,79), 7,14 (DS 1,81), 7,09 (DS 1,61) para PEG con $<8$ h, PEG con $>8$ h, $\mathrm{PSCM}$ con $<8 \mathrm{~h}, \mathrm{PSCM}>8 \mathrm{~h}$, respectivamente, siendo el primer grupo (PEG con $<8 \mathrm{~h}$ de preparación) con mejor media en BBPS, pero sin tener una diferencia estadísticamente significativa $(\mathrm{p}=0,185)$.

Tampoco hubo diferencias estadísticamente significativas en la medición de otros indicadores de calidad colonoscópica tales como intubación cecal $(\mathrm{p}=0,934)$, detección de adenomas $(\mathrm{p}=0,534) \mathrm{y}$ detección de adenocarcinomas, con uno en cada rama $(\mathrm{p}=0,653)$ (Tabla 4$)$.

Al evaluar la tolerabilidad, en la escala Likert y aplicando prueba de Mann-Whitney, se obtuvo una diferencia significativa $(\mathrm{p}=0,0004)$ a favor del PSCM. El resultado en promedio de cada una de las variables de esta escala se ve en Figura 4. De éstas, es importante destacar el sabor desagradable, náuseas, hinchazón abdominal y calambres, todas a favor del PSCM con significancia estadística. Al preguntar por ausencia laboral, no hubo diferencias entre los grupos $(p=0,260)$. En la pregunta referente a si utilizaría la misma preparación en futura colonoscopia, el grupo preparado con PSCM mostró mayor porcentaje de respuestas positivas $(p=0,002)$. En la escala EVA, la media con PEG fue 7,68 (DS 2,44 ) y con PSCM fue 9,04 (DS 1,59), con una gran diferencia estadísticamente significativa a favor del PSCM (p<0,0001) (Tabla 5).

\section{Discusión}

Este es el primer ensayo clínico nacional que compara el polietilenglicol y picosulfato de sodio más citrato de magnesio como agentes de preparación colonoscópicos.

Nuestros resultados muestran que el PEG y el PSCM son comparables en términos de efectividad de limpieza colónica aplicando el BBPS, siendo la
Tabla 2. Características basales

\begin{tabular}{|lccc|}
\hline & \multicolumn{2}{c}{ Rama de estudio } & \\
Variable & PEG & PSCM & Valor p \\
Edad (media) años & $49,09 \pm 14,64$ & $47,36 \pm 14,49$ & 0,895 \\
Sexo femenino (\%) & $61,78 \%$ & $62,12 \%$ & 0,964 \\
IMC & $25,69 \pm 3,82$ & $24,93 \pm 3,05$ & 0,254 \\
ASA (I-II) (\%) & $98,37 \%$ & $96,96 \%$ & 0,712 \\
Indicación colonoscopia* [n (\%)] & & & 0,536 \\
Sangrado gastrointestinal & $26(10,7 \%)$ & $22(20 \%)$ & \\
Cambio hábito intestinal & $37(17,2 \%)$ & $21(19 \%)$ & \\
Dolor abdominal & $18(8,3 \%)$ & $11(10 \%)$ & \\
Historia familiar CCR & $15(6,9 \%)$ & $6(5,4 \%)$ & \\
Screening CCR & $71(33 \%)$ & $28(25,4 \%)$ & \\
Vigilancia pólipo & $12(5,5 \%)$ & $5(4,5 \%)$ & \\
Seguimiento posdiverticulitis & $5(2,3 \%)$ & $4(3,6 \%)$ & \\
Otras & $31(14,4 \%)$ & $13(11,8 \%)$ & \\
\hline
\end{tabular}

CCR: Cáncer colorrectal. *Puede ser más de una indicación.

Tabla 3. Puntaje Boston de PEG y PSCM

\begin{tabular}{|lccc|}
\hline \multicolumn{3}{|c|}{ Rama de estudio } & \\
Variable & PEG & PSCM & Valor p \\
BBPS continua (media $\pm \mathrm{ds})$ & $7,51 \pm 1,66$ & $7,12 \pm 1,71$ & 0,1116 \\
BBPS categórica n $(\%)$ & & & \\
Mal preparado & $20(16,26)$ & $17(25,75)$ & 0,1167 \\
Bien preparado & $103(83,73)$ & $49(74,24)$ & \\
Tiempo entre término preparación y colonoscopia (media) & \\
$>8 \mathrm{~h}$ & $7,06 \pm 1,79$ & $7,09 \pm 1,61$ & \multirow{2}{*}{0,185} \\
\hline $8 \mathrm{~h}$ & $7,98 \pm 1,38$ & $7,14 \pm 1,81$ & \\
\hline
\end{tabular}

Tabla 4. Intubación cecal y detección neoplasia con PEG y PSCM

\begin{tabular}{|lcccc|}
\hline \multicolumn{1}{|l}{ Característica n (\%) } & \multicolumn{1}{c}{$\begin{array}{c}\text { Rama de estudio } \\
\text { PEG }\end{array}$} & PSCM & Valor p \\
\hline Intubación cecal & & & & \\
& Sí & $119(96,74)$ & $64(96,96)$ & 0,934 \\
& No & $4(3,26)$ & $2(3,04)$ & \\
Detección adenomas & & $34(27,64)$ & $16(24,24)$ & 0,534 \\
& Sí & $89(73,36)$ & $50(75,75)$ & \\
& No & $1(0,81)$ & $1(1,52)$ & 0,653 \\
\hline Detección adenocarcinoma & Sí & $122(99,19)$ & $65(98,48)$ & \\
\hline
\end{tabular}




\section{ARTÍCULO ORIGINAL}

Figura 4. Resultado de promedios de variables evaluadas en escala de Likert en cuestionario de Lawrence para PEG y PSCM.

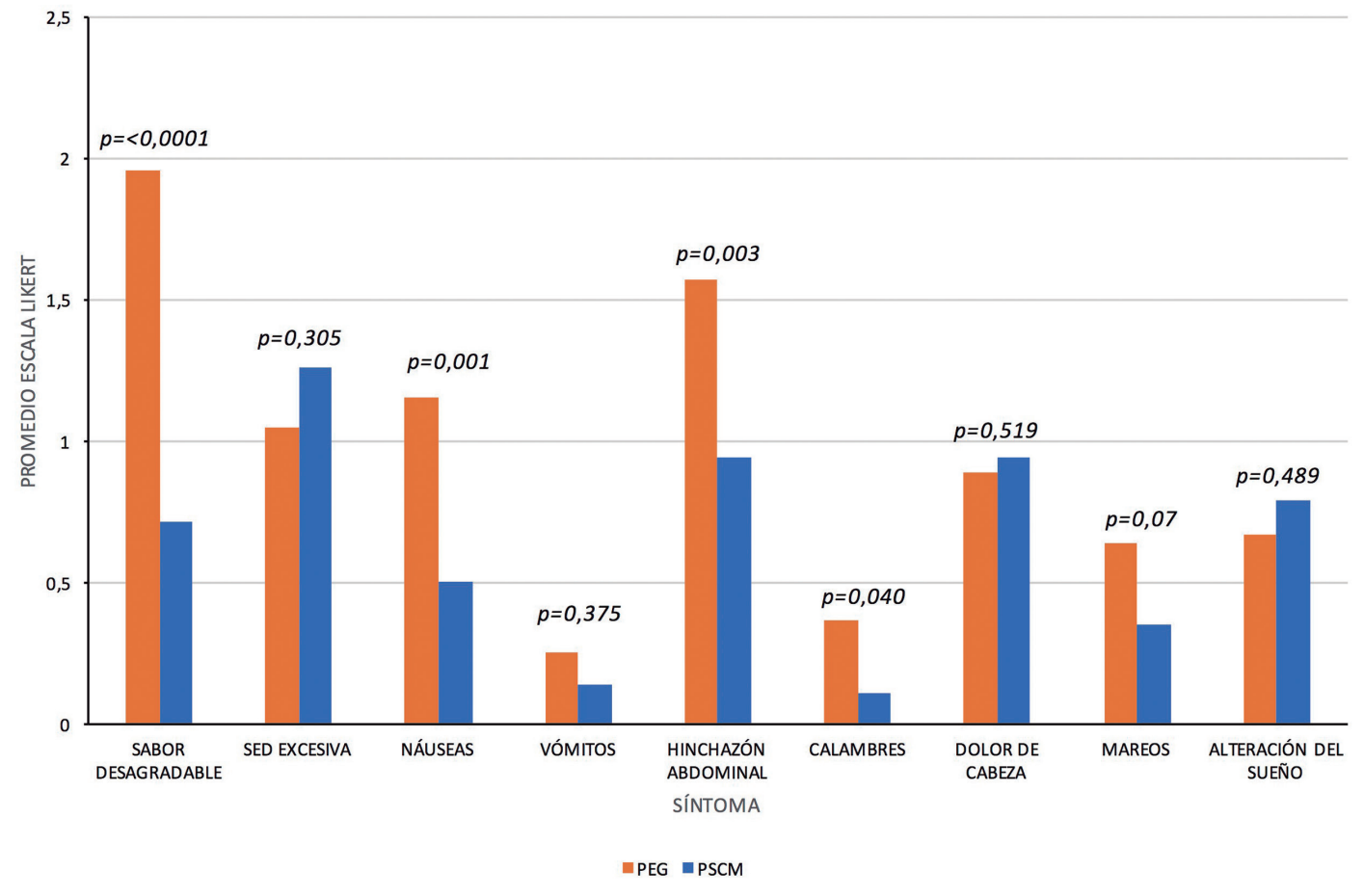

Tabla 5. Cuestionario de tolerabilidad de Lawrence para PEG y PSCM

\begin{tabular}{|c|c|c|c|}
\hline \multirow[b]{2}{*}{ Variable } & \multicolumn{2}{|c|}{ Rama de estudio } & \multirow[b]{2}{*}{ Valor $\mathbf{p}$} \\
\hline & PEG & PSCM & \\
\hline Promedio encuesta (media \pm DS) & $0,94 \pm 0,68$ & $0,63 \pm 0,61$ & 0,0004 \\
\hline \multicolumn{4}{|c|}{ ¿Ud. tuvo que ausentarse al trabajo debido a la preparación intestinal? n (\%) } \\
\hline Sí & $51(41,46)$ & $33(50)$ & \multirow{2}{*}{0,260} \\
\hline No & $72(58,53)$ & $33(50)$ & \\
\hline \multicolumn{4}{|c|}{$\begin{array}{l}\text { Si Ud. tuviera que realizarse una nueva colonoscopia en el futuro } \\
\text { ¿volvería a ocupar la misma preparación intestinal? n (\%) }\end{array}$} \\
\hline Sí & $81(65,85)$ & $57(77,27)$ & \multirow{2}{*}{0,002} \\
\hline No & $42(34,15)$ & $9(13,63)$ & \\
\hline Escala Visual Análoga (media \pm ds) & $7,68 \pm 2,44$ & $9,04 \pm 1,59$ & 0,0003 \\
\hline
\end{tabular}

tolerabilidad superior al utilizar PSCM, resultados similares con otros ensayos clínicos europeo ${ }^{17,20} \mathrm{y}$ metaanálisis ${ }^{28}$.

Si consideramos el BBPS como variable continua, el PEG muestra una tendencia a ser superior al PSCM en media de puntaje BBPS (7,51 $\pm 1,66$ vs 7,12 $\pm 1,71$, hecho que es concordante al dicotomizarla como variable categórica (bien preparado 83,73\% PEG vs 74,24\% PSCM), sin embargo, en ambos casos sin significancia estadística, pero creemos que esto es importante al considerar las recomendaciones actuales de calidad colonoscópicas que sugieren un $85 \%$ de preparación adecuada en pacientes ambulatorios ${ }^{7}$. Utilizamos la escala de Boston, ya que es una escala validada y ampliamente divulgada en la literatura y en estudios con metaanálisis que comparan diferentes escalas, muestran que ésta es la mejor para medir calidad de limpieza colónica ${ }^{23}$.

De igual forma ambos agentes son comparables 
al evaluar otras características de medición de calidad colonoscópica como lo son intubación cecal y detección de adenomas, dato que es concordante al revisar literatura internacional ${ }^{15}$.

Al medir tolerabilidad con el cuestionario de Lawrence el PSCM es, significativamente, superior al PEG en todos los aspectos que mide esta encuesta, excepto en la pregunta referente al ausentismo laboral en que no existe diferencia. En las variables evaluadas en escala Likert, el PSCM es mejor tolerado en todas ellas, excepto en sed excesiva, dolor de cabeza y alteración del sueño, sin embargo, estas últimas sin significancia estadística. En relación a sabor desagradable, náuseas, hinchazón abdominal y calambres, el PSCM es considerablemente mejor, hecho que se corrobora con el valor $\mathrm{p}<0,05$.

Sabemos que una inadecuada preparación puede conducir a pasar por alto lesiones colónicas ${ }^{29}$; ante esta relevante consecuencia, importantes sociedades científicas sugieren que la eficacia debe ser una prioridad por sobre la tolerabilidad ${ }^{5}$. Sin embargo, la eficacia y la tolerabilidad están estrechamente interrelacionadas, ya que la eficacia del agente limpiador, está influenciada por la tolerabilidad a la ingesta del agente. Una mala tolerabilidad conduce a una disconformidad del paciente afectando el grado de limpieza colónica.

Nuestro estudio tiene varias limitaciones. Por una parte, en lo referente a la muestra, ya que es de un solo centro $y$, por otro lado, los pacientes son principalmente sanos y jóvenes (media IMC 25,42, ASA I-II 97\%, media edad 48,49), dejando fuera del análisis a pacientes con comorbilidades como insuficiencia renal, hepática y cardiaca, por lo cual nuestros resultados no se pueden generalizar y aplicar a poblaciones de alto riesgo. Además, debemos considerar la variabilidad existente inter e intraobservador para el cálculo del BBPS, la cual no consideramos en el análisis de los resultados, a pesar de que los endoscopistas están entrenados en la medición del BBPS. Por otro lado, el cuestionario de Lawrence está validado al inglés, no así al español, sin embargo, sus preguntas son fácilmente traducibles al español, ya que los conceptos utilizados no son equívocos. Hubo una pérdida considerable de pacientes en la rama del PSCM, creando una asimetría en el número total de pacientes por rama, esto debido a factores exógenos, tales como cancelación de colonoscopia, negativa de completar encuesta cuando previamente ya habían aceptado participar en el estudio y toma de agente de preparación intestinal diferente al asignado, sin embargo, también tiene fortalezas, en cuanto a que se completó el número necesario de pacientes en ambas ramas y que ambos grupos son homogéneos y comparables al revisar sus características basales expuestas en Tabla 2 .

\section{Conclusión}

A la luz de nuestros resultados podemos afirmar que el PEG y el PSCM son comparables en términos de efectividad de preparación intestinal aplicando criterios de calidad colonoscópica mundialmente considerados como lo son el BBPS, intubación cecal y detección de adenomas. En términos de tolerabilidad considerando cuestionario de Lawrence, el PSCM es claramente superior al PEG.

\section{Responsabilidades éticas}

Protección de personas y animales. Los autores declaran que los procedimientos seguidos se conformaron a las normas éticas del comité de experimentación humana responsable y de acuerdo con la Asociación Médica Mundial y la Declaración de Helsinki.

Confidencialidad de los datos. Los autores declaran que han seguido los protocolos de su centro de trabajo sobre la publicación de datos de pacientes.

Derecho a la privacidad y consentimiento informado. Los autores han obtenido el consentimiento informado de los pacientes y/o sujetos referidos en el artículo. Este documento obra en poder del autor de correspondencia.

\section{Financiación}

Los autores declaran no haber recibido ninguna financiación para la realización de este trabajo.

\section{Conflicto de intereses}

Los autores declaran no tener ningún conflicto de intereses.

\section{Agradecimientos}

Los autores agradecen al staff de endoscopistas y enfermeras de Unidad de Endoscopia de Clínica INDISA, por su colaboración en el desarrollo de este estudio. 


\section{Bibliografía}

1. American Cancer Society. Cancer Facts \& Figures 2017. Atlanta: American Cancer Society; 2017. p. 1-71.

2. SEER Cancer Stat Facts: Colon and Rectum Cancer. National Cancer Institute. Bethesda, MD [citado el 10 de noviembre de 2017] Disponible desde: https://seer. cancer.gov/statfacts/html/colorect.html.

3. Departamento de Estadísticas e Información en Salud, Ministerio de Salud, Gobierno de Chile [Internet]. Santiago, Chile: Ministerio de Salud [citado el 10 de noviembre de 2017]. Disponible desde http://www.deis.cl/ wp-content/uploads/2017/08/Seriedefunciones-y-mortalidad-observadapor-tumores-malignos-edad_sexo.Chile-1997-2015.xlsx;

4. Zin T, Min Swe K, Maw M. Bowel preparation for colonoscopy (Protocol).

Cochrane Database Syst Rev. 2011;10:1-8.

5. Johnson DA, Barkun AN, Cohen LB, Dominitz JA, Kaltenbach T, Martel $\mathrm{M}$, et al. Optimizing adequacy of bowel cleansing for colonoscopy: Recommendations from the U.S. MultiSociety Task Force on Colorectal Cancer. Gastrointest Endosc. 2014;80:543-62.

6. Beck DE. Bowel preparation for colonoscopy. Clin Colon Rectal Surg. 2010;23:10-3

7. Brunner KT, Calderwood AH. Quality in Colonoscopy. Curr Gastroenterol Rep. 2015;17:1-7.

8. Ruiz-Romero D, Téllez-Ávila FI. Preparación para colonoscopia en 2016: recomendaciones actuales utilizando datos nacionales. Endoscopia 2016;28:81-9.

9. Sulz MC, Kröger A, Prakash M, Manser CN, Heinrich H, Misselwitz B. Meta-analysis of the effect of bowel preparation on adenoma detection: Early adenomas affected stronger than advanced adenomas. PLoS One. 2016;11:1-17.

10. Belsey J, Crosta C, Epstein O, Fischbach W, Layer P, Parente F, et al. Metaanalysis: The relative efficacy of oral bowel preparations for colonoscopy 1985-2010. Aliment Pharmacol Ther. 2012;35:222-37.

11. Tan JJY, Tjandra JJ. Which is the optimal bowel preparation for colonoscopy. A meta-analysis. Color Dis. 2006;8:247-58.

12. Hoy SM, Scott LJ, Wagstaff AJ. Sodium picosulfate/magnesium citrate: A review of its use as a colorectal cleanser. Drugs 2009;69:123-36.

13. Manes G, Amato A, Arena M, Pallotta S, Radaelli F, Masci E. Efficacy and acceptability of sodium picosulphate/ magnesium citrate $v s$ low-volume polyethylene glycol plus ascorbic acid for colon cleansing: A randomized controlled trial. Color Dis. 2013;15:1145-53.

14. Munsterman ID, Cleeren E, van der Ploeg T, Brohet R, van der Hulst R. "PicoBello-Klean study". Eur J Gastroenterol Hepatol. 2015;27:29-38.

15. Jeon SR, Kim HG, Lee JS, Kim JO, Lee $\mathrm{TH}$, Cho JH, et al. Randomized controlled trial of low-volume bowel preparation agents for colonic bowel preparation: 2-L polyethylene glycol with ascorbic acid versus sodium picosulfate with magnesium citrate. Int J Colorectal Dis. 2015;30:251-8.

16. Leitao K, Grimstad T, Bretthauer M, Holme Ø, Paulsen V, Karlsen L, et al. Polyethylene glycol vs sodium picosulfate/ magnesium citrate for colonoscopy preparation. Endosc Int Open [Internet]. 2014;2(4):E230-E234. Available from: http://www.thieme-connect.de/DOI/ DOI?10.1055/s-0034-1377520.

17. Muñoz-Navas M, Calleja JL, Payeras G, Herves AJ, Abreu LE, Orive V, et al. A randomized trial to compare the efficacy and tolerability of sodium picosulfate-magnesium citrate solution vs $4 \mathrm{~L}$ polyethylene glycol solution as a bowel preparation for colonoscopy. Int J Colorectal Dis. 2015;30:1407-16.

18. Kim ES, Lee WJ, Jeen YT, Choi HS, Keum B, Seo YS, et al. A randomized, endoscopist-blinded, prospective trial to compare the preference and efficacy of four bowel-cleansing regimens for colonoscopy. Scand J Gastroenterol. 2014;49:871-7.

19. Lee SW, Bang CS, Park TY, Suk KT, Baik GH, Kim DJ. Split-dose Bowel Preparation for Colonoscopy: 2 Liters Polyethylene Glycol with Ascorbic Acid versus Sodium Picosulfate versus Oral Sodium Phosphate Tablets. Korean J Gastroenterol. 2017;70:89.

20. Choi HS, Chung JW, Lee JW, Lim MY, Park DK, Kim YJ, et al. Polyethylene glycol plus ascorbic acid is as effective as sodium picosulfate with magnesium citrate for bowel preparation: A randomized trial. J Dig Dis. 2016;17:268-
73.

21. Kim HG, Huh KC, Koo HS, Kim SE, Kim JO, Kim T Il, et al. Sodium picosulfate with magnesium citrate (SPMC) plus laxative is a good alternative to conventional large volume polyethylene glycol in bowel preparation: A multicenter randomized single-blinded trial. Gut Liver 2015;9:494-501.

22. Lai EJ, Calderwood AH, Doros G, Fix OK, Jacobson BC. The Boston Bowel Preparation Scale: A valid and reliable instrument for colonoscopyoriented research. Gastrointest Endosc. 2009;69:620-5.

23. Parmar R, Martel M, Rostom A, Barkun AN. Validated Scales for Colon Cleansing: A Systematic Review. Am J Gastroenterol. 2016;111:197-204.

24. Lawrence IC, Willert RP, Murray K. A validated bowel-preparation tolerability questionnaire and assessment of three commonly used bowel-cleansing agents. Dig Dis Sci. 2013;58:926-35.

25. World Medical Association. World Medical Association Declaration of Helsinki. Ethical Principles for Medical Research Involving Human Subjets. Jama 2013;310:2191-4.

26. Asociación Médica Mundial. Declaración de Taipei sobre Consideraciones Éticas de las bases de datos de salud y Biobancos. Asociación médica mundial [Internet]. Ferney-Voltaire, Francia [citado el 10 de agosto de 2017]. Disponible desde: https://www.wma.net/es/policies-post/ declaracion-de-la-amm-sobre-lasconsideraciones-eticas-de-las-bases-dedatos-de-salud-y-los-biobancos/

27. Cobos-Carbó A, Augustovski F. Declaración CONSORT 2010: actualización de la lista de comprobación para informar ensayos clínicos aleatorizados de grupos paralelos. Med Clin (Barc). 2011;137:213-5.

28. Jin Z, Lu Y, Zhou Y, Gong B. Systematic review and meta-analysis: Sodium picosulfate/magnesium citrate $v s$ polyethylene glycol for colonoscopy preparation. Eur J Clin Pharmacol. 2016;72:523-32.

29. Harewood GC, Sharma VK, De Garmo $\mathrm{P}$. Impact of colonoscopy preparation quality on detection of suspected colonic neoplasia. Gastrointest Endosc. 2003;58:76-9. 